\title{
Práticas Educativas no Contexto da Maternidade Adolescente: Breve Revisão da Literatura
}

\author{
Angela Helena Marin \\ Universidade Luterana do Brasil \\ Daniela Centenaro Levandowski \\ Universidade do Vale do Rio dos Sinos
}

\begin{abstract}
RESUMO
A idade materna tem sido apontada como um importante fator de influência sobre as práticas educativas. Este artigo consiste em uma revisão da literatura nacional e internacional, publicada entre os anos de 1986 a 2006, sobre as práticas educativas comumente utilizadas por mães adolescentes com filhos pequenos. Grande parte dos estudos revisados salientou o uso de práticas educativas coercitivas por essas mães. Contudo, também foram encontrados estudos que evidenciaram que, apesar da idade, mães adolescentes cuja rede de apoio social é efetiva tendem a utilizar práticas educativas menos coercitivas. Além disso, outras variáveis, como escolaridade, nível socioeconômico, conhecimento sobre o desenvolvimento infantil, depressão e estresse, também apareceram como fatores de influência sobre o uso e a adequação das práticas educativas por essas mães. Frente à inconsistência desses achados, sugere-se que novas pesquisas empíricas sejam levadas a cabo, principalmente no contexto brasileiro, para permitir uma melhor caracterização das mães adolescentes em relação a esse aspecto.
\end{abstract}

Palavras-chave: práticas educativas; adolescência; maternidade.

\begin{abstract}
Childrearing Practices in the Context of Adolescent Motherhood: Brief Literature Review

Maternal age has been pointed to as an important influence factor on childrearing practices. This article addresses a revision of the national and international literature published between 1986 and 2006 about childrearing practices used by adolescent mothers of toddlers. A great part of the reviewed studies pointed out the use of coercive childrearing practices by adolescent mothers. However, studies were also identified revealing that, in spite of their age, adolescent mothers with adequate social support tend to use less coercive childrearing practices. Other variables, such as maternal education, socioeconomic level, knowledge about child development, depression, and stress, emerged as influence factors in the use and adaptation of childrearing practices by adolescent mothers. New empirical researches examining this inconsistency need to be conducted in a Brazilian context to allow for a better characterization of adolescent mothers' childrearing.
\end{abstract}

Keywords: childrearing practices; adolescent motherhood.

O contexto familiar é reconhecido como um fator importante para o desenvolvimento infantil. Conforme Darling e Steinberg (1993), a influência deste contexto pode se dar a partir de dois modos distintos: os estilos parentais e as práticas educativas. Os estilos parentais podem ser definidos como o conjunto de atitudes dos pais direcionadas à criança, as quais criam um clima emocional em que o comportamento parental é expresso. Já as práticas educativas são estratégias utilizadas pelos pais para atingir objetivos específicos em termos de comportamento e características da criança.
Existem diferentes concepções teóricas acerca das estratégias que os pais utilizam na educação de seus filhos, mas, para fins do presente artigo, será utilizada a perspectiva de Hoffman $(1975,1994)$, por esta ser uma das mais destacadas pela literatura atual. As práticas educativas, conforme Hoffman, são concebidas a partir do modo como os pais orientam os comportamentos da criança, o qual pode ser classificado em indutivo ou explicativo e coercitivo ou punitivo. As práticas indutivas ou explicativas caracterizam-se por atingir o objetivo disciplinar, indicando à criança as conseqüências do seu comportamento para as outras 
pessoas, ao invés das conseqüências punitivas para ela mesma. Isso propicia à criança a compreensão das implicações de suas ações e, portanto, dos motivos que justificam a necessidade de mudança do seu comportamento. Já as práticas coercitivas ou punitivas caracterizam-se pela aplicação direta da força e do poder dos pais, incluindo punição física, privação de privilégios e afeto ou uso de ameaças destas atitudes. Essas estratégias fazem com que a criança controle seu comportamento em função das reações punitivas dos pais. Além disso, elas produzem emoções intensas, como medo, raiva e ansiedade, que tendem a reduzir a possibilidade de compreensão da situação e a necessidade de modificação do comportamento por parte da criança (Hoffman, 1975, 1994).

Visando a avaliar como os pais costumam agir com seus filhos, as pesquisas têm buscado contemplar o contexto em que os comportamentos parentais ocorrem. Alguns fatores têm sido destacados como influentes para o uso e a adequação das práticas educativas parentais, os quais podem ser enquadrados em três grandes categorias, de acordo com Belsky (1984): o contexto social, os atributos da criança e as características pessoais dos pais. Entre estas últimas, destacase a idade, em especial, a idade da mãe, uma vez que a maioria dos estudos prioriza a investigação das práticas educativas maternas. Essa ênfase pode ser explicada pelo fato de a mãe ainda ser apontada como tendo um papel educativo mais marcante do que o pai e por ser a maior responsável pelo cuidado e supervisão dos filhos (Brook, Zheng, Whiteman \& Brook, 2001; Costa, Teixeira \& Gomes, 2000; Weber, Viezzer \& Brandenburg, 2004). Assim, é plausível supor que as práticas educativas maternas tendem a ter maior impacto no desenvolvimento da criança do que as práticas educativas paternas.

Nesse sentido, surpreende o fato de serem raros os estudos sobre as práticas educativas utilizadas por mães adolescentes, apesar da grande incidência de gestações nesta faixa etária, tanto no Brasil como em outros países (Hoyert, Mathews, Menacker, Strobino \& Guyer, 2006) e da idade materna ser indicada como um importante fator de influência sobre o uso das práticas educativas (Davis-Kean, 2005; Kelley, Power $\&$ Wimbush, 1992). Por conseguinte, o presente estudo teve como objetivo realizar uma revisão da literatura nacional e internacional publicada entre os anos de 1986 e 2006 sobre as práticas educativas utilizadas por mães adolescentes com filhos pequenos.
Para tanto, foi feita uma busca nas bases de dados Scielo, utilizando-se como descritores os termos "maternidade" e "adolescente". Foi localizado apenas um artigo nacional que se referia ao foco deste estudo. Já na base de dados PsycInfo, utilizou-se, inicialmente, os termos "práticas educativas" (childrearing practices) e "mães adolescentes" (adolescent mothers), considerando-se como critérios de restrição: o período de publicação do trabalho (1986-2006), o tipo de publicação (journal), o idioma (inglês) e a população (humanos). Através desse procedimento, foram recuperados 73 registros. Destes, foram excluídos: os artigos que não foram localizados na íntegra (apenas com resumo disponível) e cujo periódico não estava acessível; os que não focalizavam o tema do presente estudo, como, por exemplo, aqueles sobre práticas educativas de mães e pais com filhos adolescentes, de pais adolescentes ou de avós; os que travavam de outros tipos de práticas, como as alimentares; e aqueles que enfocavam as práticas utilizadas por mães adolescentes com filhos em idade escolar ou as expectativas das gestantes adolescentes frente ao uso de tais práticas após o nascimento do bebê. Foram excluídos ainda artigos que se referiam a comentários sobre outros trabalhos já publicados sobre o tema e aqueles que utilizaram amostras de populações aborígines ou com retardo mental. Após esta seleção, 19 artigos foram efetivamente considerados para análise.

Em um segundo momento, foram acrescentados os termos "técnicas ou estratégias educativas" (childrearing techniques/strategies) em combinação ao termo "adolescent mothers", tendo sido recuperado um registro, que já havia sido considerado na busca anterior. Com os termos "técnicas ou estratégias disciplinares" (disciplinary techniques/strategies), combinados com "adolescent mothers", não foram recuperados registros.

$\mathrm{Na}$ tentativa de localizar outros estudos relacionados ao tema, foi feita uma busca em periódicos impressos, sendo encontrados mais dois estudos. Assim, contou-se com 22 artigos (21 internacionais e um nacional) para análise, os quais serão apresentados a seguir.

\section{PRÁTICAS EDUCATIVAS MATERNAS NO CONTEXTO DA MATERNIDADE ADOLESCENTE}

A idade materna foi apontada como um importante fator de influência no uso e adequação das práticas educativas por Fox, Platz e Bentley (1995), a partir de uma investigação com 1.056 mães norte-americanas de crianças de um a quatro anos, que responderam a 
instrumentos padronizados que avaliavam disciplina, afeto/cuidado, expectativas em relação ao desenvolvimento da criança e problemas emocionais ou comportamentais infantis. Os resultados indicaram que o uso de práticas coercitivas era mais freqüente entre mães solteiras adolescentes do que entre mães solteiras adultas, mantidas semelhantes as demais características sócio-demográficas, como etnia e nível socioeconômico. Utilizando a mesma amostra e instrumentos do estudo referido, Brenner e Fox (1999) constataram que, entre as famílias de menor nível educacional e socioeconômico, as mães mais jovens puniam mais suas crianças e esperavam que elas pudessem fazer mais do que seriam capazes, com base no seu nível de desenvolvimento, em comparação às mães adultas que apresentavam igual condição.

Nessa mesma direção, o estudo de Reis, BarberaStein e Bennett (1986), realizado com 210 mães norteamericanas de 15 a 24 anos, que foram avaliadas em relação à depressão, ao apoio social, às atitudes de cuidado da criança e ao conhecimento sobre o desenvolvimento infantil e ao ambiente familiar, revelou que as mães jovens ( 20 a 24 anos) tendiam a ser menos punitivas e criavam ambientes mais apoiadores aos seus filhos/as, em comparação às mães adolescentes (15 a 19 anos). Contudo, os autores ressaltaram como fatores associados à coercitividade materna, além da idade, o menor conhecimento sobre o desenvolvimento infantil, uma rede de apoio social pouco efetiva e índices elevados de depressão.

Da mesma forma, Reis (1988), a partir de uma amostra de 696 mães norte-americanas, que também responderam a um instrumento que investigava as atitudes de cuidado da criança e o conhecimento sobre o desenvolvimento infantil, ressaltou que aquelas que tinham 16 anos ou menos apresentavam maiores dificuldades de compreensão do desenvolvimento infantil e práticas educativas mais inadequadas para com a criança, quando comparadas com mães mais velhas (com idade superior a 17 anos). Tais achados corroboram os resultados do estudo conduzido por Camp (1995), que comparou 106 mães adolescentes (menores de 18 anos) com 47 mães adultas (maiores de 19 anos) norte-americanas, através de questionários autoadministrados sobre práticas educativas. $\mathrm{O}$ autor indicou o uso mais freqüente de práticas coercitivas pelas adolescentes.

O mesmo havia sido encontrado em um estudo realizado por Reis (1989), comparando um grupo de 150 mães adolescentes jovens (16 anos ou menos), 260 mães adolescentes (17 a 19 anos) e 242 mães adultas (20 a 25 anos), todas norte-americanas. Instrumentos que avaliavam depressão, apoio social, atitudes de cuidado da criança e o conhecimento sobre o desenvolvimento infantil foram aplicados. Foi possível constatar que as mães adolescentes mais jovens apresentaram-se mais coercitivas com suas crianças, em comparação às demais mães adolescentes e às mães adultas. É importante salientar, no entanto, que as mães desse primeiro grupo também apresentaram índices mais elevados de depressão.

De forma paradoxal, embora grande parte dos estudos revisados tenha salientado implicações negativas da maternidade adolescente, também foram localizados estudos que não apontaram diferenças quanto ao uso das práticas educativas em função da idade materna. Por exemplo, destaca-se o estudo longitudinal realizado por Benasich e Brooks-Gunn (1996), que acompanhou 608 crianças norte-americanas prematuras e suas mães desde o nascimento até os três anos de vida da criança, levando em consideração, entre outras variáveis, as sócio-demográficas, o conhecimento e conceitos de práticas educativas maternas, a qualidade do ambiente familiar e o desenvolvimento cognitivo e os problemas de comportamento da criança. Os autores indicaram, entre outros achados, que o uso de práticas educativas não variou em função da idade da mãe.

Também Luster e Rhoades (1989) encontraram semelhanças entre dois grupos de mães norte-americanas brancas, 20 adolescentes (15-20 anos) e 20 adultas (21-35 anos), quanto às crenças e práticas educativas. Instrumentos padronizados que avaliavam a qualidade do ambiente familiar, as crenças parentais sobre as práticas educativas e sobre a influência do ambiente no desenvolvimento, bem como a percepção parental do temperamento infantil, foram empregados. As participantes referiram utilizar com maior freqüência práticas educativas indutivas, que consideravam mais apropriadas ao desenvolvimento infantil.

Comparando longitudinalmente (do primeiro ao décimo segundo mês de vida do bebê), 19 mães adolescentes com 25 mães adultas norte-americanas, por meio de medidas de atitudes e crenças sobre a maternagem e o cuidado da criança e da qualidade da maternagem em termos de características do ambiente físico e da interação mãe-bebê, Baranowski, Schilmoeller e Higgins (1990) encontraram menor empatia em relação às necessidades da criança entre as mães adolescentes, em comparação às adultas. Nos demais aspectos avaliados, não foram encontradas diferenças significativas entre os grupos, embora as adolescentes te- 
nham geralmente obtido menores escores. Além disso, aquelas adolescentes com expectativas desenvolvimentais mais adequadas, maior empatia e menos crenças positivas quanto à punição corporal tenderam a fornecer um ambiente doméstico de melhor qualidade e a se engajar em comportamentos mais positivos em relação à criança.

A despeito dos estudos que realizaram comparações entre mães adolescentes e adultas, também foram localizadas pesquisas que tiveram como foco apenas mães adolescentes, mas que apontaram para alguns resultados semelhantes aos já mencionados, no que diz respeito ao maior uso de práticas educativas coercitivas. Por exemplo, no contexto nacional, o estudo de Silva e Salomão (2003) retratou, a partir de entrevistas, a percepção de 25 mulheres sobre suas filhas que se tornaram mães na adolescência, revelando a imaturidade e a impaciência das jovens para lidarem com o bebê. Foi destacada a presença de violência física, através de atitudes como bater, empurrar e afastar a criança de si mesma, para não ouvir seu choro. Já no contexto internacional, Crockenberg (1987), examinando 40 mães adolescentes norte-americanas (17 a 21 anos), cujos filhos estavam com 24 meses de idade, através de uma avaliação do temperamento e do desenvolvimento da criança, do ambiente doméstico, da interação mãe-criança, da história de desenvolvimento da própria jovem e do apoio social recebido, constatou que elas demonstravam mais raiva e comportamentos coercitivos para com a criança quando haviam experienciado rejeição na própria infância e recebido pouco apoio do parceiro após o parto. Ressalta-se que esse tipo de comportamento materno não esteve associado à irritabilidade da criança.

Como visto, os estudos revisados indicaram que se tornar mãe na adolescência parece aumentar a probabilidade de adotar comportamentos e atitudes coercitivas com relação à criança. Cabe destacar que, no extremo desta tendência, foram localizados inclusive estudos que associaram a maternidade adolescente ao maltrato infantil, em particular o abuso físico e/ou a negligência (Klerman, 1993; Murphy, Gilliland \& Griswold-Rhymer, 2001). Dentre os fatores apontados pela literatura para a ocorrência desses comportamentos encontram-se: ter sido vítima de abuso físico (Paúl \& Domenech, 2000), apresentar estresse psicológico, menor nível de realização acadêmica e insatisfação com o apoio social recebido (Budd, Heilman \& Kane, 2000). Contudo, é importante mencionar que as pesquisas que investigaram a associação entre a idade materna e o maltrato infantil apresentaram achados contraditórios, sugerindo que outros fatores individuais e até mesmo contextuais seriam importantes para determinar o risco de sua ocorrência.

Frente ao exposto, é interessante ressaltar que alguns estudos localizados levaram em conta fatores que tenderiam a diminuir o uso de práticas educativas coercitivas pelas mães adolescentes. Dentre esses fatores, o apoio social foi bastante destacado. Por exemplo, Bunting e McAuley (2004), a partir de uma revisão da literatura norte-americana e inglesa, verificaram que o apoio familiar e do parceiro exerciam uma influência positiva sobre as práticas educativas e os comportamentos maternos das adolescentes. De igual modo, McCullough e Sherman (1998), que avaliaram uma amostra de 107 mães de 14 a 21 anos que faziam parte de programas de treinamento para pais, sugeriram que oferecer à mãe adolescente um ambiente de aceitação e apoio poderia ajudá-la a construir uma identidade positiva como mãe e, assim, a utilizar menos freqüemtemente práticas educativas coercitivas ou punitivas. Para tanto, os autores utilizaram instrumentos padronizados que objetivavam obter informações das mães adolescentes sobre os programas e serviços de saúde oferecidos e avaliar o ambiente familiar e a ocorrência de abuso físico.

Resultados semelhantes foram encontrados em um estudo conduzido por Weis (2002) com 234 mães adolescentes negras norte-americanas, avaliando depressão, eficácia no desempenho do papel materno, apoio social, problemas de comportamento da criança e qualidade do ambiente doméstico. Aquelas mães adolescentes que demonstravam mais amor, atenção e afeto, ao invés de raiva ou controle coercitivo, recebiam maior apoio familiar no cuidado diário da criança e apresentaram diversos fatores de proteção durante a adolescência, como morar com os pais e permanecer estudando.

Os achados do estudo de Uno, Florsheim e Uchino (1998) também reforçam tais proposições, uma vez que mães adolescentes (14 a 19 anos) norte-americanas de descendência mexicana e caucasiana, cujos filhos tinham entre um e três anos de idade, apresentavam comportamentos mais afetivos quando estavam empregadas, vivenciavam níveis moderados de estresse e contavam com uma rede de apoio social efetiva. Tais participantes foram avaliadas quanto à percepção de apoio social, estresse global e parental, expectativas quanto ao comportamento infantil e uso de disciplina e afeto. 
Ainda corroborando tais achados, Strassberg e Treboux (2000), a partir de uma investigação conduzida em duas fases, com uma amostra inicial de 43 díades mães-criança norte-americanas, avaliando táticas de conflito e coerção materna, problemas de comportamento da criança e a resposta emocional da mãe a imagens de crianças, indicaram que fatores contextuais, tais como contatos sociais aversivos e relação conjugal estressante, faziam com que as mães adolescentes decodificassem uma variedade de expressões emocionais da criança como indicativas de raiva, e atribuíssem mais intenções de desobediência/desafio aos comportamentos infantis, reagindo a isso com maior coercitividade.

Também SmithBattle (2000), avaliando longitudinalmente (por oito anos) 16 mães adolescentes norte-americanas e suas famílias, para investigar as repercussões das tradições familiares de cuidado no desenvolvimento do cuidado pela mãe adolescente, através de entrevistas, encontrou como um fator de influência nas práticas de cuidado dessas mães os modelos familiares. Em alguns casos, tal modelo foi repetido (apesar das tentativas e desejo de mudança), pela falta de apoio e de exemplos positivos alternativos. Em outros casos, foi modificado pela descoberta de novos modelos, especialmente da família do parceiro, pelo apoio dele recebido e pela possibilidade de reflexão decorrente de tratamento psicoterápico. Nesse sentido, o autor reforçou a necessidade de investigação das experiências, emoções, história, hábitos e contexto social na organização das preocupações, habilidades e aprendizagens dessas mães.

Por sua vez, McKenry, Kotch e Browne (1991), investigaram o uso de práticas coercitivas em 157 mães adolescentes norte-americanas, aos 12 meses da criança, bem como diversos aspectos pessoais (percepção de rejeição da própria mãe, depressão, autoestima, habilidades de coping, eventos de vida negativos), contextuais (nível de pobreza, apoio social e estresse), da relação mãe-criança (qualidade da relação, expectativas quanto ao desenvolvimento infantil, crenças quanto à utilização da punição corporal, responsividade, empatia) e da criança (peso, temperamento e nível de desenvolvimento). Os autores identificaram os recursos pessoais e psicológicos das mães como os melhores preditores para o uso dessas práticas coercitivas, em comparação às fontes contextuais de estresse e apoio social. Mais especificamente, baixa auto-estima, etnia negra e não ser casada, associados à presença de eventos de vida altamente estressantes e redes de apoio restritas apareceram como importantes preditores no uso de punição corporal.
A partir da exposição dos estudos revisados, foi possível constatar que em sua maioria eles eram de caráter empírico (91\%) e quantitativo (91\%), utilizando instrumentos de medida padronizados para a avaliação de diferentes aspectos relacionados às práticas educativas, tais como depressão, apoio social, qualidade do ambiente familiar, conhecimento e expectativa sobre o desenvolvimento infantil, crenças sobre a educação dos filhos, entre outros. Destaca-se que apenas um dos estudos utilizou a observação da interação mãe-criança como instrumento de avaliação das práticas educativas. Alguns deles focalizaram apenas a avaliação da mãe $(62 \%)$ sobre as práticas educativas utilizadas, enquanto outros investigaram concomitantemente aspectos da mãe e da criança (48\%), como o seu temperamento ou desenvolvimento, a fim de detectar possíveis correlações entre comportamento materno e características infantis. Além disso, ficou evidente que os estudos foram predominantemente realizados com população norte-americana $(90 \%)$, retratando eventuais peculiaridades daquele contexto. Quanto ao delineamento utilizado, prevaleceram os estudos transversais $(86 \%)$ e houve uma equivalência entre os estudos comparativos (adolescentes versus adultas) e aqueles que focalizaram apenas as mães adolescentes. Nesse caso, cabe destacar que os instrumentos utilizados em geral foram validados para a utilização com mães e pais adultos, não se tendo conhecimento sobre sua validade junto a mães adolescentes.

\section{CONSIDERAÇÕES FINAIS}

O presente estudo, que teve como objetivo efetuar uma revisão da literatura nacional e internacional, publicada entre 1986 e 2006, quanto às práticas educativas no contexto da maternidade adolescente, constatou uma inconsistência entre os achados dos artigos revisados quanto ao tipo de prática educativa predominantemente utilizada por essas mães. Enquanto alguns estudos indicaram diferenças entre as práticas educativas utilizadas por mães adolescentes e adultas, ou entre as próprias adolescentes, no sentido de maior coercitividade, outros estudos não apontaram nessa direção. Essas diferenças podem ser explicadas, em parte, como uma conseqüência indireta da presença de variáveis sócio-demográficas desfavoráveis entre as adolescentes ou até mesmo da inadequação dos instrumentos utilizados para a avaliação.

Assim, para que não se confundam as influências da idade materna e de outras variáveis sobre o uso de práticas educativas, é importante que os estudos reali- 
zados com mães adolescentes levem em consideração alguns indicadores sócio-demográficos, que, quando pareados, podem reduzir ou até mesmo eliminar as diferenças encontradas entre adolescentes e adultas (Klerman, 1993). Também se destaca que qualquer diferença percebida poderá estar refletindo mais a diversidade social e econômica do que efeitos decorrentes exclusivamente da idade (Paúl \& Domenech, 2000). Mais do que isso, a comparação entre mães adolescentes e adultas pode vir a mascarar a grande variabilidade na qualidade do cuidado parental provido pelas mães de cada um desses grupos, nem sempre adequadamente avaliada nos estudos revisados.

Desse modo, concorda-se com SmithBattle (2000), que ressalta que, embora a maternidade na adolescência venha sendo bastante estudada, os delineamentos transversais e o foco nos achados deficitários adotados pela maioria dos estudos da área têm exagerado as conseqüências negativas de uma gravidez precoce. Para o autor, tais estudos têm obscurecido como a maternidade na adolescência pode ser considerada um rito de passagem para a idade adulta, particularmente na ausência de recursos e aspirações escolares ou ocupacionais. De fato, as circunstâncias sociais das mães adolescentes parecem influenciar mais efetivamente a forma como elas fazem uso das práticas educativas do que a idade propriamente dita. Portanto, uma rede de apoio familiar e social efetiva pode ajudá-las a interpretar adequadamente as necessidades de seus filhos, a fazer uso de práticas educativas indutivas, disponibilizar afeto e, conseqüentemente, a utilizar com menor freqüencia práticas educativas coercitivas.

Diante disso, pontua-se a necessidade de se abandonar o modelo "doentio" da maternidade adolescente, conforme destacam Baranowski e colaboradores (1990), pois, apesar de se veicular um perfil da adolescente em situação de risco, alguns estudos acabam encontrando poucas diferenças entre elas e as mães adultas e outros, nenhuma, como foi o caso de alguns aqui revisados. Para os autores, melhor seria especificar os aspectos que necessitam ser reforçados em uma intervenção junto a mães adolescentes, levando em conta as suas necessidades e dificuldades, o que também foi apontado por Seitz e Apfel (1999) a partir de uma revisão da literatura sobre a eficácia de alguns programas destinados a melhorar as práticas de cuidado infantil no contexto norte-americano.

De qualquer forma, programas de intervenção podem ser planejados para reduzir a eventual tendência ao uso de práticas maternas coercitivas entre adolescentes, servindo como uma fonte de apoio para elas
(Seitz \& Apfel, 1999). Para tanto, baseado nas idéias de Weis (2002), considera-se oportuno ensinar técnicas parentais não coercitivas para disciplinar a criança, acessar e transformar as crenças mal adaptativas sobre educação e auxiliar na redução do nível de estresse psicológico dessas jovens, quando presente. Contudo, a fim de se planejar intervenções efetivas e eficazes, sugere-se que novos estudos abordem o tema das práticas educativas no cenário da maternidade adolescente, especialmente no contexto brasileiro. Tais estudos necessitam investigar amplamente os fatores associados a elas, em diferentes momentos no tempo e de forma quantitativa e qualitativa. Mais do que isso, deveriam empregar instrumentos validados também para a população adolescentes.

\section{REFERÊNCIAS}

Baranowski, M. D., Schilmoeller, G. L., \& Higgins, B. S. (1990). Parenting attitudes of adolescent and older mothers. Adolescence, 2, 781-790.

Belsky, J. (1984). The determinants of parenting: A process model. Child Development, 55, 83-96.

Benasich, A. A., \& Brooks-Gunn, J. (1996). Maternal attitudes and knowledge of child-rearing: Associations with family and child outcomes. Child Development, 67, 1186-1205.

Brenner, V., \& Fox, R. A. (1999). An empirically derived classification of parenting practices. Journal of Genetic Psychology, 160, 343-356.

Brook, J. Zheng, L., Whiteman, M., \& Brook, D. W. (2001). Aggression in toddlers: Associations with parenting and marital relations. Journal of Genetic Psychology, 162, 228-241.

Budd, K. S., Heilman, N. E., \& Kane, D. (2000). Psychosocial correlates of child abuse potential in multiply disadvantaged adolescent mothers. Child Abuse \& Neglect, 24, 611-625.

Bunting, L., \& McAuley, C. (2004). Research review: Teenage pregnancy and motherhood: The contribution of support. Child and Family Social Work, 9, 207-215.

Camp, B. W. (1995). Maternal characteristics of adolescent mothers and older mothers of infants. Psychological Reports, 77(3), 1152-1154.

Costa, F. T., Teixeira, M. A. P., \& Gomes, W. B. (2000). Responsividade e exigência: Duas escalas para avaliar estilos parentais. Psicologia: Reflexão e Crítica, 13, 465-473.

Crockenberg, S. (1987). Predictors and correlates of anger toward and punitive control of toddlers by adolescent mothers. Child Development, 58, 964-975.

Darling, N., \& Steinberg, L. (1993). Parenting style as a context: An integrative model. Psychological Bulletin, 113, 487-496.

Davis-Kean, P. E. (2005). The influence of parent education and family income on child achievement: The indirect role of parental expectations and the home environment. Journal of Family Psychology, 19, 294-304.

Fox, R. A., Platz, D. L., \& Bentley, K .S. (1995). Maternal factors related to parenting practices, developmental expectations, and perceptions of child behavior problems. Journal of Genetic Psychology, 156, 431-441. 
Hoffman, M. L. (1975). Moral internalization, parental power, and the nature of parent-child interaction. Developmental Psychology, 11, 228-239.

Hoffman, M. L. (1994). Discipline and internalization. Developmental Psychology, 30, 26-28.

Hoyert, D. L., Mathews, T. J., Menacker, F., Strobino, D. M., \& Guyer, B. (2006). Annual summary of vital statistics: 2004. Pediatrics, 117(1), 168-183.

Kelley, M. L., Power, T. G., \& Wimbush, D. D. (1992). Determinants of disciplinary practices in low-income Black mothers. Child Development, 63, 573-582.

Klerman, L. V. (1993). The relationship between adolescent parenthood and inadequate parenting. Children and Youth Services Review, 15, 309-320.

Luster, T., \& Rhoades, K. (1989). The relation between childrearing beliefs and the home environment in a sample of adolescent mothers. Family Relations: Journal of Applied Family and Child Studies, 38, 317-322

McCullough, M., \& Sherman, A. (1998). Family of origin interaction and adolescent mother's potential for child abuse. Adolescence, 33(130), 375-384.

McKenry, P. C., Kotch, J. B., \& Browne, D. H. (1991). Correlates of dysfunctional parenting attitudes among low-income adolescent mothers. Journal of Adolescent Research, 6(2), 212-234.

Murphy, L. M., Gilliland, K. M., \& Griswold-Rhymer, H. (2001). Unintentional injury among very young children: Differential Risck for children of adolescent mothers? Childrens's Health Care, 30(4), 293-308.

Paúl, J., \& Domenech, L. (2000). Childhood history of abuse and child abuse potential in adolescent mothers: A longitudinal study. Child Abuse \& Neglect, 24, 701-713.

Reis, J. (1988). Child-rearing expectations and developmental knowledge according to maternal age and parity. Infant Mental Health Journal, 9, 287-304.
Reis, J. (1989). A comparison of young teenage, older teenage, and adult mothers on determinants of parenting. Journal of Psychology: Interdisciplinary and Applied, 123, 141-151.

Reis, J., Barbera-Stein, L., \& Bennett, S. (1986). Ecological determinants of parenting. Family Relations, 35, 547-554.

Seitz, V., \& Apfel, N. H. (1999). Effective interventions for adolescent mothers. Clinical Psychology: Science and Practice, 6, 50-66.

Silva, D. V., \& Salomão, N. M. R. (2003). A maternidade na perspectiva de mães adolescentes e avós maternas dos bebês. Estudos de Psicologia, 8, 135-145.

SmithBattle, L. (2000). Developing a caregiving tradition in opposition to one's past: Lessons from a longitudinal study of teenage mothers. Public Health Nursing, 17(2), 85-93.

Strassberg, Z., \& Treboux, D. (2000). Interpretations of child emotion expressions and coercive parenting practices among adolescent mothers. Social Development, 9(1), 80-95.

Uno, D., Florsheim, P., \& Uchino, B. N. (1998). Psychosocial mechanisms underlying quality of parenting among Mexican American and white adolescent mothers. Journal of Youth and Adolescence, 27, 585-603.

Weber, L. N. D., Viezzer, A. P., \& Brandenburg, O. J. (2004). O uso de palmadas e surras como prática educativa. Estudos de Psicologia, 9, 227-237.

Weis, R. (2002). Parenting dimensionality and typology in a disadvantaged African American sample: A cultural variance perspective. Journal of Black Psychology, 28, 142-173.

Recebido: 10/04/2007

Última revisão: 06/03/2008

Aceite final: 10/05/2008

Sobre as autoras:

Angela Helena Marin: Psicóloga, mestre e doutoranda em Psicologia pela Universidade Federal do Rio Grande do Sul (UFRGS) e docente na Universidade Luterana do Brasil (ULBRA).

Daniela Centenaro Levandowski: Psicóloga, mestre e doutora em Psicologia pela Universidade Federal do Rio Grande do Sul, com Estágio Pós-Doutoral na Pontifícia Universidade Católica do Rio Grande do Sul. Docente do Programa de Pós-Graduação em Psicologia Clínica e do Curso de Psicologia da Universidade do Vale do Rio dos Sinos (Unisinos).

Endereço para orrespondência: Angela Helena Marin - Rua Garibaldi, 1254/301 - 90035-052 Porto Alegre/RS.

Endereço eletrônico: ahmarin@hotmail.com. 